\title{
Characteristics of Convergence Curve of the PE Estimate of Variance Ratio in the Balanced Case
}

\author{
Kohichi YAMAGISHI ${ }^{1,2}$ and Hiroaki IWAISAKI ${ }^{2}$ \\ ' Niigata Prefectural Kohnohkan High School, Maki-machi, Niigata-ken 953-0041, Japan \\ 2 Faculty of Agriculture, Niigata University, Niigata-shi 950-2181, Japan
}

(Received May 25, 1999 ; Accepted August 27, 1999)

\begin{abstract}
The pseudoexpectation (PE) methods are an iterative procedure for estimating variance components in the general mixed linear model. The computational loads in the PE methods are relatively small and the estimates converge faster, although the methods are an approximation to the restricted maximum likelihood (REML) procedure. In the field of animal breeding, it has been considered that the PE methods can be used in estimating from large data sets including no or weak influence of selection, or in obtaining initial values to use in the REML estimation. This paper reports somc basic characteristics of the convergence curve of the PE variance ratio estimate in the casc of balanced data. In the specific balanced case, the PE and REML estimates are identical, but their convergence curves are different. The ratios of the difference between the consecutive estimates in the $i$ th and the $i-1$ th rounds to that in the $i+1$ th and the $i$ th rounds vary, for instance, in the REML estimation using the expectation-maximization algorithm, while those with the PE methods take a constant value regardless of initial values and rounds of iteration. Hence, the PE curve of convergence can be exactly represented using the result for a geometric series. Consequently, the PE curve is equivalent to the curve assumed underlyingly in the extrapolation techniques, or the techniques of directly predicting the final value such as the Aitken extrapolation and the common intercept approach. In the unbalanced case, the propcrty of this kind of the PE curve is no longer retained, and the degrees of discrepancies among the PE and the extrapolation estimates would be dependent on the structure of the data to be analyzed. When one wishes to accelerate convergence in the REML analysis of unbalanced data, it is important to notice that the PE and the extrapolation techniques each have the advantages and disadvantages for the purpose.
\end{abstract}

Animal Science Journal 70 (6) : 414-420, 1999

Key words : Pseudoexpectation method, Estimate of variance ratio, Convergence curve, Balanced data, Extrapolation technique

Of the methods for estimating variance components in mixed linear models from unbalanced data, the pseudoexpectation (PE) method of Schaeffer ${ }^{17)}$ developed in 1986 is an extension of Thompson's procedure $^{19)}$ to deal with the situations of multiple components of variance. The Thompson procedure is a corrected version of Cunningham and Henderson's procedure ${ }^{2)}$ that was proposed as a modified version of Henderson's Method III' ${ }^{5)}$. The quadratic forms in Method III do not depend on the variance components functionally, while those in the $\mathrm{PE}$ methods including the other two variants ${ }^{6,20)}$ are no longer free of the components of variance. Thus, a process of iterative estimation is required with the $\mathrm{PE}$ methods, as is done with the restricted maximum likelihood (REML) procedure ${ }^{12}$.

The PE methods are classified into one type of the REML-like procedures. They are actually approximations to REML that mimic the computational steps in the REML procedure using the expectation-

Corresponding : Hiroaki IWAISAKI (fax : +81 (0) 25-262-6854, e-mail : iwsk@agr.niigata-u.ac.jp)

Anim. Sci. J. 70 (6) : 414-420, 1999 
maximization algorithm (EM-REML) ${ }^{3,4)}$ in different degrees $^{6,9,17,18,20)}$ and can handle relatively large data sets. In animal breeding applications, it has been considered that the PE methods can be useful in estimating variance components in large populations with no or weak selection or in obtaining the preassigned values to use in the REML estimation for large selected populations ${ }^{1,6,11,18,20)}$. The use of the PE approaches for the latter purpose may be justified and valid, since it has been observed in numerical or simulation studies ${ }^{1,6,11,20)}$ that PEs give the estimates akin to the REML estimate at a relatively rapid rate of convergence with less computational requirements. Since it would be not easy to treat unbalanced cases analytically, assuming balanced data, Yamagishi and Iwaisaki $^{21)}$ proved a certain performance for the rapid convergence of the PE methods relative to the EMREML procedure.

In this paper, considering a balanced data-design, additional basic properties of the PE methods for convergence and their relations to the extrapolation techniques of directly predicting the final converged value are investigated.

\section{Theoretical Consideration}

\section{Model and the PE estimate}

Consider a mixed linear model

$$
\mathbf{y}=\mathbf{X} \beta+\mathbf{Z u}+\mathbf{e},
$$

where $y$ is the vector of observations, $\beta$ is the vector of fixed effects, $u$ is the vector of random effects, $e$ is the vector of random residuals, and $\mathbf{X}$ and $\mathbf{Z}$ are the incidence matrices, assuming a balanced, crossclassified design with no covariances among elements of the random vectors. With this model, it is supposed here that $\mathrm{E}(\mathbf{u})$ and $\mathrm{E}(\mathbf{e})$ are null, $\mathrm{E}(\mathbf{y})=\mathbf{X} \beta$, $\operatorname{Var}(\mathbf{u})=\mathbf{I} \sigma_{u}^{2}, \operatorname{Var}(\mathbf{e})=\mathbf{I} \sigma_{e}^{2}$, and $\operatorname{Cov}\left(\mathbf{u}, \mathbf{e}^{\prime}\right)=\mathbf{0}$, result ing in $\operatorname{Var}(\mathbf{y})=\mathbf{Z} \mathbf{Z}^{\prime} \sigma_{u}^{2}+\mathbf{I} \sigma_{c}^{2}$. Denoting the number of levels for the random factor by $s$, the number of observations per level of the random factor by $t$, and the total number of observations by $\mathrm{N}$, we have

$$
\hat{\mathbf{u}}=(\mathrm{t}+\alpha)^{-1} \mathbf{Z}^{\prime} \mathbf{M y},
$$

where $\hat{\mathbf{u}}$ is the solution to $\mathbf{u}$ obtained from the empirical mixed model equations using a priori value of the variance ratio $\alpha=\tilde{\sigma}_{\mathrm{c}}^{2} / \tilde{\sigma}_{\mathrm{u}}^{2}$, and $\mathbf{M}$ is the projection matrix describing the absorption of the fixed effects.
For all the PE methods ${ }^{6,17,20)}$, we then obtain

$$
\hat{\sigma}_{\mathrm{u}}^{2}=\mathrm{b}\{(\mathrm{s}-1) \mathrm{t}(\mathrm{t}+\alpha)\}^{-1}
$$

and

$$
\hat{\sigma}_{e}^{2}=\left\{a-b(t+\alpha)^{-1}\right\}_{c^{-1}}
$$

with $a=\mathbf{y}^{\prime} \mathbf{M y}, \quad b=\mathbf{y}^{\prime} \mathbf{M Z Z} \mathbf{M y}$ and $c=\mathbf{N}-\mathbf{r}(\mathbf{X})$, where $\mathbf{r}(\mathbf{X})$ is the rank of $\mathbf{X}$, since the data are assumed to be balanced.

Under the condition $\alpha_{i}>0$, where $i$ is the iteration number, therefore, we find

$$
\alpha_{i+1}=b_{1} \alpha_{i}+b_{0}\left(1>b_{1}>0 ; b_{0}>0\right)
$$

with

$$
b_{0}=(a t-b)(b c)^{-1}(s-1) t
$$

and

$$
b_{1}=a(b c)^{-1}(s-1) t
$$

as discussed by Yamagishi and Iwaisaki ${ }^{21)}$.

Differences between two consecutive estimates and their ratios

Let the 'difference' be

$$
\mathrm{d}_{i}=\alpha_{i+1}-\alpha_{i}
$$

Then, using equation (5), we have

$$
\mathrm{d}_{i}=\mathrm{g}_{1} \alpha_{i}+\mathrm{g}_{0}
$$

where

$$
\begin{aligned}
& \text { and } \begin{aligned}
& \mathrm{g}_{0}=\mathrm{b}_{0} \\
& \mathrm{~g}_{1}=\{\mathrm{a}(\mathrm{s}-1) \mathrm{t}-\mathrm{bc}\}(\mathrm{bc})^{-1} \\
& \text { Moreover, for the ratios }\left(\mathrm{r}_{i}\right) \text { of } \mathrm{d}_{i-1} \text { to } \mathrm{d}_{i} \text {, we obtain } \\
& \mathrm{r}_{i}=\{\mathrm{a}(\mathrm{s}-1) \mathrm{t}\}^{-1} \mathrm{bc} \\
&=\mathrm{r}
\end{aligned}
\end{aligned}
$$

Therefore, it is seen that the ratios are exactly a constant for any estimates of the variance ratio in the iterative process.

In contrast, $d_{i}$ in the case of EM-REML are expressed as

$$
\mathrm{d}_{i}=\sum_{j=1}^{2} \mathbf{h}_{1 \mathrm{j}} \alpha_{i}^{\mathrm{j}}\left\{\sum_{k=0}^{2} \mathbf{h}_{2 \mathrm{k}} \alpha_{i}^{\mathrm{k}}\right\}^{-1}
$$

with

$$
\begin{aligned}
& \mathrm{h}_{11}=(a t-b)(s-1) t \\
& h_{12}=a(s-1) t-b c \\
& h_{20}=(a t-b) t \\
& h_{21}=a(s+1) t+b(c-s)
\end{aligned}
$$

and

$$
\mathrm{h}_{22}=\text { as. }
$$

Hence, the ratios $\mathbf{r}_{i}\left(=\mathrm{d}_{i-1} / \mathrm{d}_{i}\right)$ are given as

$$
\mathbf{r}_{i}=\phi_{1}\left(\alpha_{i-1}\right) \phi_{2}\left(\alpha_{i}\right)\left\{\phi_{1}\left(\alpha_{i}\right) \phi_{2}\left(\alpha_{i-1}\right)\right\}^{-1} \text {, }
$$
where 

and

$$
\phi_{1}\left(\alpha_{i}\right)=\{\mathrm{a}(\mathrm{s}-1) \mathrm{t}-\mathrm{bc}\} \alpha_{i}^{2}+(\mathrm{at}-\mathrm{b})(\mathrm{s}-1) \mathrm{t} \alpha_{i},
$$

$$
\begin{aligned}
\phi_{2}\left(\alpha_{i}\right)= & \text { as } \alpha_{i}^{2}+\{\mathrm{a}(\mathrm{s}+1) \mathrm{t}+\mathrm{b}(\mathrm{c}-\mathrm{s})\} \alpha_{i} \\
& +(\mathrm{at}-\mathrm{b}) \mathrm{t} .
\end{aligned}
$$

Thus, as stated by Misztal and Schaeffer ${ }^{10)}, r_{i}$ in EMREML vary over iterations.

Convergence pattern and converged value

Noticing equation (8), $\alpha_{i}$ in the case of PE can exactly be rewritten as

$$
\alpha_{i}=\alpha_{0}+\mathrm{d}_{0}\left(\sum_{j=0}^{i-l} \mathrm{r}^{-j}\right),
$$

using a geometric series, where $\alpha_{0}$ denotes an initial value. Then we have

$$
\lim _{i \rightarrow \infty} \alpha_{i}=\alpha_{0}+\mathrm{d}_{0}\left(1-\mathrm{r}^{-1}\right)^{-1} .
$$

Consequently, from equation (12), we obtain the converged value $\left(\alpha_{c}\right)$, as follows

$$
\alpha_{c}=(a t-b)(s-1) \mathrm{t}\{-\mathrm{a}(\mathrm{s}-1) \mathrm{t}+\mathrm{bc}\}^{-1} \text {. }
$$

Now, replacing $\alpha_{i}$ by $\alpha$, define $\mathrm{R}$ as the ratio of $\mathrm{d}_{i}$ in EM-REML to that in PE, as follows

$$
\mathrm{R}=\mathbf{w}_{10} \alpha\left(\sum_{\mathrm{k}=0}^{2} \mathbf{w}_{2 \mathrm{k}} \alpha^{\mathrm{k}}\right)^{-1}
$$

where

$$
\begin{aligned}
& \mathbf{w}_{10}=b c \\
& \mathbf{w}_{20}=(a t-b) t \\
& \mathbf{w}_{21}=a(a+1) t+b(c-s)
\end{aligned}
$$

and

$$
\mathbf{w}_{22} \text {-as. }
$$

Then, assuming the normal convergence, all the coefficients, $w_{10}$ and $w_{2 k}$, are positive. We further find $w_{21}>w_{10}$, since $a t-b>0$ and $s>2$. Therefore, we have

$$
0<\mathbf{R}<1
$$

with

$$
\begin{aligned}
\lim _{\alpha \rightarrow \infty} R & =\lim _{\alpha \rightarrow \infty} w_{10}\left(\sum_{k=0}^{2} w_{2 k} \alpha^{k-1}\right)^{-1} \\
& =0 .
\end{aligned}
$$

These findings show that the 'difference' in PE is always larger than that in EM-REML and if $\alpha$ be very large, then the 'difference' in EM-REML will relatively be very small, indicating that the $P E$ estimate converges faster than the EM-REML estimate.

\section{Relations to some extrapolation techniques}

Consider two different initial values in the PE estimation, or $\alpha_{01}$ and $\alpha_{02}$. Then, from equation (12), the converged value can be written as

$$
\alpha_{c}=\alpha_{01}+\mathrm{d}_{01}\left(1-\mathrm{r}^{-1}\right)^{-1}
$$

and also as

$$
\alpha_{\mathrm{c}}=\alpha_{02}+\mathrm{d}_{02}\left(1-\mathrm{r}^{-1}\right)^{-1}
$$

\begin{tabular}{|c|c|c|c|c|c|c|c|}
\hline \multirow{2}{*}{$\begin{array}{l}\text { Animal } \\
\text { number }\end{array}$} & \multicolumn{2}{|c|}{ Level } & \multirow{2}{*}{ Observation } & \multirow{2}{*}{$\begin{array}{l}\text { Animal } \\
\text { number }\end{array}$} & \multicolumn{2}{|c|}{ Level } & \multirow{2}{*}{ Observation } \\
\hline & Fixed & Random & & & Fixed & Random & \\
\hline 1 & 1 & 1 & 16.9 & 11 & 2 & 3 & 2.2 \\
\hline 2 & 1 & 1 & 18.9 & 12 & 2 & 3 & 3.1 \\
\hline 3 & 2 & 1 & 8.6 & 13 & 1 & 4 & 9.2 \\
\hline 4 & 2 & 1 & 8.4 & 14 & 1 & 4 & 13.7 \\
\hline 5 & 1 & 2 & 14.2 & 15 & 2 & 4 & 7.5 \\
\hline 6 & 1 & 2 & 12.3 & 16 & 2 & 4 & 10.3 \\
\hline 7 & 2 & 2 & 1.0 & 17 & 1 & 5 & 14.4 \\
\hline 8 & 2 & 2 & 5.8 & 18 & 1 & 5 & 23.0 \\
\hline 9 & $\perp$ & 3 & 15.4 & 19 & 2 & 5 & 7.0 \\
\hline 10 & 1 & 3 & 15.2 & 20 & 2 & 5 & 4.3 \\
\hline
\end{tabular}

with $\mathrm{d}_{01}=\alpha_{11}-\alpha_{01}$ and $\mathrm{d}_{02}=\alpha_{12}-\alpha_{02}$. Therefore, from these two equations, we straightforwardly obtain

$$
\alpha_{\mathrm{c}}=\left(\alpha_{01} \mathrm{~d}_{02}-\alpha_{02} \mathrm{~d}_{01}\right)\left(\mathrm{d}_{02}-\mathrm{d}_{01}\right)^{-1} \text {. }
$$

Equation (16) is exactly the converged value given by the common intercept approach (CIA) ${ }^{16}$.

Furthermore, let $\alpha_{i}, \alpha_{i+1}$ and $\alpha_{i+2}$ represent three consccutive estimates in the PE iteration. Then we have

$$
\alpha_{c}=\alpha_{i}+\mathrm{d}_{i}\left(1-\mathrm{r}^{-1}\right)^{-1}
$$

and

$$
\alpha_{\mathrm{c}}=\alpha_{i+1}+\mathrm{d}_{i+1}\left(1-\mathbf{r}^{-1}\right)^{-1} .
$$

Table 1. A data-sample for the numerical illustration 
Table 2. Convergence patterns of the estimate of variance ratio for the EM-REML procedure ${ }^{1)}$

\begin{tabular}{|c|c|c|c|c|c|}
\hline Initial value & Iteration no. & $\alpha_{i}$ & $\alpha_{i+1}$ & $\mathrm{~d}_{i}$ & $\mathbf{r}_{i}$ \\
\hline \multirow[t]{12}{*}{3} & 0 & 3.0 & 3.339370 & .339370 & - \\
\hline & 1 & 3.339370 & 3.629031 & .289661 & 1. 171610 \\
\hline & 2 & 3.629031 & 3.877736 & .248705 & 1. 164677 \\
\hline & 3 & 3.877736 & 4.092405 & .214668 & 1. 158556 \\
\hline & 4 & 4.092405 & 4.278540 & .186135 & 1. 153294 \\
\hline & 5 & 4. 278540 & 4. 440564 & .162025 & 1.148806 \\
\hline & $\vdots$ & $\vdots$ & $\vdots$ & $\vdots$ & $\vdots$ \\
\hline & 50 & 5.655962 & 5.656800 & .000838 & 1. 119468 \\
\hline & $\vdots$ & $\vdots$ & $\vdots$ & $\vdots$ & $\vdots$ \\
\hline & 100 & 5.663788 & 5.663791 & .000003 & 1. 119323 \\
\hline & $\vdots$ & $\vdots$ & & & \\
\hline & $151^{2)}$ & 5.663816 & & & \\
\hline \multirow[t]{12}{*}{10} & 0 & 10.0 & 9.642414 & -.357586 & - \\
\hline & 1 & 9.642414 & 9.307971 & -.334444 & 1.069196 \\
\hline & 2 & 9.307971 & 8.996006 & -.311964 & 1.072057 \\
\hline & 3 & 8.996006 & 8. 705778 & -.290229 & 1.074892 \\
\hline & 4 & 8.705778 & 8.436470 & -.269308 & 1.077683 \\
\hline & 5 & 8.436470 & 8.187207 & -.249263 & 1.080417 \\
\hline & $\vdots$ & $\vdots$ & $\vdots$ & $\vdots$ & $\vdots$ \\
\hline & 50 & 5.685108 & 5. 682842 & -.002267 & 1. 118929 \\
\hline & $\vdots$ & $\vdots$ & $\vdots$ & $\vdots$ & $\vdots$ \\
\hline & 100 & 5.663892 & 5. 663884 & -.000008 & 1. 119321 \\
\hline & $\vdots$ & $\vdots$ & & & \\
\hline & $160^{22}$ & 5.663816 & & & \\
\hline
\end{tabular}

${ }^{1)}$ See text for notations.

${ }^{2)}$ Converged under the given criterion.

Thus, we find

$$
\alpha_{\mathrm{c}}=\left(\alpha_{i} \alpha_{i+2}-\alpha_{i+1}^{2}\right)\left(\alpha_{i+2}-2 \alpha_{i+1}+\alpha_{i}\right)^{-1}, \quad \text { (17) }
$$
because $\mathrm{d}_{i}=\alpha_{i+1}-\alpha_{i}$ and $\mathrm{d}_{i+1}=\alpha_{i+2}-\alpha_{i+1}$. Consequently, equation (17) is just the formula for Aitken's approximation $(\mathrm{AA})^{13)}$.

\section{Illustrative Example}

We give a simple numerical illustration, using a small data-sample as listed in Table 1. For the sample, note that $s=5, t=4$ and $N=20$. Fitting the model (1), the data were analyzed by the EM-REML and PE procedures using two different initial values, or $\alpha_{01}=3$ and $\alpha_{02}=10$. Convergence was assumed when change of the estimate between two consecutive rounds of iteration was less than $10^{-6} \%$.

The results for the EM-REML and PE estimation are shown in Tables 2 and 3, respectively. EMREML required 151 and 160 rounds of iteration to attain convergence for initial values of 3 and 10 , respectively. The converged value was 5.663816 . Note that as shown before, this value can also be obtained directly from equation (13), since the example data are balanced. On the other hand, one can see that with PE, the numbers of iterations necessitated to reach convergence were much lower than those for EM-REML. That is, the numbers for PE are 
YAMAGISHI and IWAISAKI

Table 3. Convergence patterns of the estimate of variance ratio for the PE procedure ${ }^{1)}$

\begin{tabular}{|c|c|c|c|c|c|}
\hline Initial value & Iteration no. & $\alpha_{i}$ & $\alpha_{i+1}$ & $\mathrm{~d}_{i}$ & $\mathbf{r}_{i}$ \\
\hline \multirow[t]{8}{*}{3} & 0 & 3.0 & 3.857573 & .857573 & 一 \\
\hline & 1 & 3.857573 & 4. 439064 & .581491 & 1. 474783 \\
\hline & 2 & 4. 439064 & 4.833353 & .394289 & 1. 474783 \\
\hline & 3 & 4.833353 & 5.100708 & .267354 & 1. 474783 \\
\hline & 4 & 5.100708 & 5. 281991 & .181284 & 1. 474783 \\
\hline & 5 & 5.281991 & 5. 404914 & .122922 & 1.474783 \\
\hline & $\vdots$ & $\vdots$ & & & \\
\hline & $48^{23}$ & 5.663816 & & & \\
\hline \multirow[t]{8}{*}{10} & 0 & 10.0 & 8. 604035 & -1.395965 & - \\
\hline & 1 & 8.604035 & 7. 657478 & -.946556 & 1. 474783 \\
\hline & 2 & 7.657478 & 7.015651 & -.641828 & 1. 474783 \\
\hline & 3 & 7.015651 & 6. 580449 & -.435202 & 1. 474783 \\
\hline & 4 & 6.580449 & 6. 285354 & -.295095 & 1. 474783 \\
\hline & 5 & 6.285354 & 6.085260 & -.200094 & 1. 474783 \\
\hline & $\vdots$ & $\vdots$ & & & \\
\hline & $49^{2)}$ & 5.663816 & & & \\
\hline
\end{tabular}

1) See text for notations.

2) Converged under the given criterion.

approximately one-thirds, compared to those for EMREML.

As expected from the definition, for both the iterative methods, the absolute values of $d_{i}$ decrease towards zero, as the process of iterative estimation proceeds. With EM-REML, it is seen that according to initial values, $r_{i}$ vary over iterations. Namely, $r_{i}$ gradually decrease and increase over iterations, when $\alpha_{0}<\alpha_{c}$ and $\alpha_{0}>\alpha_{c}$, respectively. Contrary to this fact, we see that with $P E, r_{i}$ take a constant value over iterations, or 1.474783 in the current case, irrespectively of initial values. It should be noticed for the PE procedure that the converged value of 5.663816 can be yielded by equation (12).

For CIA, with $\alpha_{01}=3$ and $\alpha_{02}=10$, having $\mathrm{d}_{01}=$ .857573 and $\mathrm{d}_{02}=-1.395965$ with $\mathrm{PE}$, equation (16) leads to 5.663816 , or the converged value. In contrast, in the case of EM-REML, we have $d_{01}=.339370$ and $d_{02}=-.357586$, and then the value obtained from CIA becomes 6.408524 .

For AA, when we use a set of the first three consecutive estimates with $\mathrm{PE}$, or $3.857573,4.439064$ and
4.833353, equation (17) gives the value of 5.663816 . However, use of the corresponding estimates with EM-REML, namely, 3.339370, 3.629031 and 3.877736 , in equation (17) results in 5.387994 .

\section{Discussion}

Yamagishi and Iwaisaki ${ }^{21)}$ described the relationships between $\alpha_{i+1}$ and $\alpha_{i}$ for the Tilde-Hat ${ }^{20)}$ and EM-REML ${ }^{4)}$ procedures, assuming the balanced data. Noticing their formulae [13] and [16] and equating $\alpha_{i+1}$ to $\alpha_{i}$, we also obtain equation (13) in this paper for both the procedures. Hence, in the balanced case, these iterative methods give the same point of convergence for the estimates. Moreover, as discussed by Yamagishi and Iwaisaki ${ }^{21}$, the PE and REML estimates are identical to the conventional ANOVA and MIVQUE estimates ${ }^{7.8,14,15)}$, when converged within the parameter space.

As has been shown in this study, however, the convergence patterns of the PE and EM-REML estimates are different from each other, even if the converged values are the same. In the case of EM- 
REML, the ratios $r_{i}$ are dependent on $\alpha_{i}$ and $\alpha_{i-1}$, and consequently the ratio value varies over rounds of iteration. In contrast, as shown by equation (8), since the ratio $r_{i}$ in the $P E$ procedures is free of the prior value of the variance ratio, it takes a constant value. Therefore, the convergence curve for the PE approach can be exactly repesented by using the result for the sum of a geometric series.

The extrapolation techniques such as CIA and AA are a procedure for reducing the number of iterations in the iterative methods. Also, it may be used for rapidly (and approximately in unbalanced cases) determining whether the estimate(s) will converge or not. It is known that CIA is equivalent to assuming a nonlinear model to describe the convergence curve of the variance ratio estimate for an iterative method $^{10)}$. However, the CIA and AA estimates obtained in some process of REML estimation do not equal the final estimate, even in the balanced case as assumed in this study. Rather, the convergence curve which can be appropriately described by CIA and AA has been shown to be identical to the curve in the PE approaches applied to the assumed, balanced case. Then, in the balanced case, the guessed value of the final converged value based on the extrapolation techniques exactly corresponds to the converged value, when the techniques are used in the PE estimation. For this specific case, therefore, an equivalence between the PE approaches and the extrapolation techniques is established.

Contrary to this finding, the equivalence is no longer retained, when data are unbalanced. In unbalanced cases, the $P E$ procedures of three $\operatorname{kinds}^{6,17,20)}$ show different curves of convergence, and consequently their converged values are different from each other ${ }^{6,11}$. Also, the PE estimates are not equal to the guessed values obtained using the extrapolation techniques. The magnitudes of discrepancies among the $\mathrm{PE}$ and the guessed values would be dependent on the structure of the data used, for instance, the unbalancedness and the number of observations per level for the random factor.

With unbalanced data, the PE approaches and the extrapolation techniques may be useful in speeding up the convergence in the REML estimation using an algorithm such as EM. That is, the estimate obtained from a preliminary PE analysis or the guessed value from an extrapolation technique applied in the appropriate stage of REML estimation can be used as the (re-) starting value for REML estimation. Generally, it may be appropriate to apply CIA or AA, when the REML convergence curve is totally smooth and the techniques are effectively applicable, since the techniques require only one round each for two different initial values or three consecutive rounds of iteration. However, it is empirically known that the REML convergence curves are not smooth frequently and rather fluctuating in earlier rounds of iteration, even when only a few parameters are estimated. In such a case, even the AA technique could not be used, until the convergence curves become smooth. On the other hand, the PE procedures are generally applicable to such a situation, although they necessitate certain rounds of iteration to attain convergence. In accelerating convergence in the REML analysis of actual unbalanced data, it is important to notice that the PE and the extrapolation techniques each have the advantages and disadvantages for the purpose.

\section{References}

1) Bertrand JK, Kriese LA. Two methods for parameter estimation using multiple-trait models and beef cattle field data. Journal of Animal Science, $68: 2310$ $-2318.1990$.

2) Cunningham EP, Henderson CR. An iterative procedure for estimating fixed effects and variance components in mixed model situations. Biometrics, 24 : 1325. 1968

3) Dempster AP, Laird NM, Rubin DB. Maximum likelihood from incomplete data via the EM algorithm. Journal of the Royal Statistical Society [Series B], $39: 1-38.1977$.

4) Harville DA. Maximum likelihood approaches to variance component estimation and to related problems. Journal of the American Statistical Association, $72: 320-338.1977$.

5) Henderson CR. Estimation of variance and covariance components. Biometrics, $9: 226-252.1953$.

6) Iwaisaki H. A modified pseudoexpectation method for variance component estimation. Journal of Animal Breeding and Genetics, 109 : 90-97. 1992.

7) LaMotte LR. A class of estimators of variance 
components. In : Technical Report, No. 10, Dept. of Statistics, Univ. of Kentucky. Lexington. 1970.

8) LaMotte LR. Locally best quadratic estimators of variance components. In : Technical Report, No. 22, Dept. of Statistics, Univ. of Kentucky. Lexington. 1971.

9) Manfredi E, Ducrocq V, Foulley JL. Genetic analysis of dystocia in dairy cattle. Journal of Dairy Science, $74: 1715-1723.1991$.

10) Misztal I, Schaeffer LR. Nonlinear model for describing convergence of iterative methods of variance component estimation. Journal of Dairy Science, 69 : 2209-2213. 1986.

11) Ouweltjes W, Schaeffer LR, Kennedy BW. Sensitivity of methods of variance component estimation to culling type of selection. Journal of Dairy Science, $71: 773-779.1988$.

12) Patterson HD, Thompson R. Recovery of inter-block information when block sizes are unequal. Biometrika, 58 : 545-554. 1971.

13) Press WH, Flannery BP, Teukolsky SA, Vetterling WT. Numerical Recipes. 1st ed. 132-135. Cambridge Univ. Press. Cambridge. 1986.

14) Rao CR. Minimum variance quadratic unbiased estimation of variance components. Journal of Multivariate Analysis, 1:445-456. 1971.
15) Rao CR. MINQUE theory and its relation to $\mathrm{ML}$ and MML estimation of variance components. Sankhyä [Series B], $41:$ 138-153. 1979.

16) Schaeffer LR. Estimation of variance and covariance components for average daily gain and backfat thickness in swine. In : Variance Components and Animal Breeding. (Van Vleck LD, Searle SR eds.) 123-138. Cornell Univ. Ithaca. 1979.

17) Schaeffer LR. Pseudo expectation approach to variance component estimation. Journal of Dairy Science, 69 : 2884-2889. 1986.

18) Shi MJ, Laloè D, Ménissier F, Renand G. Estimation of genetic parameters of preweaning performance in the French Limousin cattle breed. Genetics Selection Evolution, 25 : 177-189. 1993.

19) Thompson R. Iterative estimation of variance components for non-orthogonal data. Biometrics, 25 : 767-773. 1969.

20) Van Raden PM, Jung YC. A general purpose approximation to restricted maximum likelihood: The tilde-hat approach. Journal of Dairy Science, $71: 187$ $-194.1988$.

21) Yamagishi K, Iwaisaki $\mathrm{H}$. Convergence property of the estimate of variance ratio in the tilde-hat procedure with balanced data. Animal Science and Technology, 69 : 341-346. 1998. 